Comparative dosimetric analysis and NTCP modelling of 4D-CT planning scans within the UK NeoSCOPE trial

Corresponding author:

Dr Owen Nicholas

South West Wales Cancer Centre

Singleton Hospital

Sketty Lane

Swansea

SA2 8QA

Email: owen.nicholas@wales.nhs.uk

Authors:

Owen Nicholas $2,3,5$, Caitlin Bowden ${ }^{1,2}$, Adam Selby ${ }^{2}$, Owen Bodger ${ }^{3}$, Paul Lewis ${ }^{3}$, Richard Webster ${ }^{1}$, Ganesh Radhakrishna ${ }^{4}$, Gareth Jones ${ }^{1,5}$, Maria Hawkins ${ }^{6}$, Somnath Mukherjee ${ }^{6}$, Tom Crosby ${ }^{1}$, Sarah Gwynne $2,3,5$

Affiliations:

1. Velindre Cancer Centre, Cardiff

2. South West Wales Cancer Centre, Swansea

3. Swansea University Medical School, Swansea

4. St James University Hospital, Leeds

5. National RTTQA Group

6. CRUK/MRC Oxford Institute for Radiation Oncology, Oxford

Keywords:

Radiotherapy, oesophageal cancer, 3D-CT planning, 4D-CT planning, post-operative toxicity, organs at risk, NTCP

Declaration:

We thank the NeoSCOPE Trials Management Group for providing us access to the radiotherapy related patient data.

Abstract: Neoadjuvant chemoradiotherapy for resectable oesophageal cancer improves overall survival compared to surgery alone but is associated with increased toxicity. NeoSCOPE is a trial of two different nCRT regimens for resectable oesophageal and was the first multi-centre trial in the UK to incorporate 4D-CT into the radiotherapy planning. Using NeoSCOPE 4D-CT cases, we undertook a dosimetric comparison study of 3D-CT versus 4D-CT plans comparing target volume coverage and dose to organs at risk. We used established 
normal tissue complication probability (NTCP) models to evaluate the potential toxicity reduction of using 4D-CT plans in oesophageal cancer. Our work shows that incorporating 4D$\mathrm{CT}$ into treatment planning may significantly reduce the toxicity burden from this treatment.

Introduction

Despite improvements in surgical techniques, outcomes from surgery for oesophageal cancer (OC) remain poor [1]. Neoadjuvant chemoradiotherapy (nCRT) is a treatment strategy that has been shown to improve outcomes, but is associated with concerns over toxicity, particularly in the post-operative setting [2]. This is in part responsible for the variable uptake of nCRT around the UK $[3,4]$.

Limiting dose to organs at risk (OARs) is postulated to reduce post-operative complications but traditionally decisions were based on dose volume histograms, in turn based on a 3D scan, which is a snapshot in time of both the tumour position, but also of OARs. Respiration (leading to tumour deformation and motion particularly in the cranio-caudal direction), swallowing, peristalsis, gastric filling, emptying and vascular/cardiac pulsations all effect the position of the tumour during treatment [5-7] and can affect the doses received by the OARs.

Two methods currently in use accounting for this motion are respiratory gating and fourdimensional CT (4D-CT) planning. The use of 4D-CT scanning has the potential to reduce the resulting risk of geographical miss, by accounting for this patient-specific variation over the course of a respiration cycle.[7] The NeoSCOPE Trial was the first multi-centre UK trial to incorporate 4D-CT into RT planning. [8]

In addition to characterising the range of motion, 4D-CT has been shown to have a dosimetric benefit in non-small cell lung cancer with Cole et al. showing that by reducing dose to OARs, 4D-CT allowed isotoxic dose escalation, with the hope that this would lead to improved local control and better overall survival. [5] It is not known to what extent these findings would apply to other thoracic tumours like the oesophagus. The aim of this study was to determine if the use of 4D-CT scans in the NeoSCOPE study resulted in any dosimetric advantage to OARs by using dose volume histogram (DVH) and established NTCP models to ascertain any potential clinically meaningful toxicity reduction. 


\section{NeoSCOPE Trial}

NeoSCOPE was a non-blinded, randomised (1:1 via a centralised computer system), 'pick a winner' phase II trial for patients with resectable oesophageal adenocarcinoma investigating the benefit of two different nCRT regimens for OC. Surgery was performed 6 to 8 weeks after nCRT. Primary end-point was pathological complete response (pCR). Secondary end-points included toxicity, surgical morbidity/mortality, resection rate and overall survival. In the NeoSCOPE trial, 30-d post-operative respiratory and cardiac complication rates were $36.6 \%$ $40 \%$ and $9.8 \%-25.7 \%$ respectively. Full trial results have been published elsewhere.[8]

Both arms in the study received 2 cycles of induction chemotherapy with Oxaliplatin $\left(130 \mathrm{mg} / \mathrm{m}^{2}\right.$ day 1$)$ and Capecitabine $\left(625 \mathrm{mg} / \mathrm{m}^{2}\right.$ days $\left.1-21\right)$. Patients were then randomized to Oxaliplatin $\left(85 \mathrm{mg} / \mathrm{m}^{2}\right.$ IV days $\left.1,18,29\right)$ and Capecitabine $(625 \mathrm{mg}$ PO BD on days of radiotherapy) with radiotherapy or carboplatin (AUC 2 ) and paclitaxel $\left(50 \mathrm{mg} / \mathrm{m}^{2} \mathrm{IV}\right.$ on days 1 , 8, 15, 22 and 29) with RT. RT consisted of $45 G y$ in 25 fractions over 5 weeks. Centres participating in the study could choose to do either 3D or 4D CT planning scans with 4D CT simulation encouraged for lower oesophagus/GEJ tumours. [8]

Gross tumour volume (GTV) was defined using diagnostic CT scan, endoscopy, EUS and PET scan (when available). The clinical target volume (CTV) was calculated by growing the GTV by $2 \mathrm{~cm}$ manually along the oesophagus superiorly, inferiorly and $1 \mathrm{~cm}$ radially, editing out lungs and bronchus, heart, liver, aorta and vertebrae. All OARs were defined as per trial protocol and delineated on a 3D-CT scan that was used for planning and radiotherapy delivery. A 3DCT scan was mandated in trial protocol irrespective of whether 4D-CT was used or not. The planning target volume (PTV) for the 3D cases (PTV3D) was created by growing CTV $1 \mathrm{~cm}$ superiorly and inferiorly and $0.5 \mathrm{~cm}$ radially. For 4D cases a PTV4D was created by growing the internal target volume (ITV) by $0.5 \mathrm{~cm}$. [9]

In order to facilitate centres to undertake 4D-CT in the trial, the RT protocol gave two options for creation of an ITV with 4D-CT (see appendix 1), reflecting the practice of two of the centres with the most experience in 4D-CT for oesophageal RT at that point in time. Centres wishing to undertake 4D-CT within the trial were encouraged to attend a workshop with break-out sessions for both physicists and clinicians, looking at issues surrounding scan acquisition and outlining respectively. A 4D-CT pre-accrual test case was also made available for those who 
were not able to attend the workshop that had to be satisfactorily completed. Eight centres were approved by the trial to use 4D-CT planning scans for lower third oesophageal tumours and these eight centres could choose whether to use a 4D-CT planning scan or not. The data used in this study is made up of 4D-CT planning scans from Oxford, Leeds and Cardiff. [8]

28/85 (33\%) patients recruited to the UK NeoSCOPE trial had a 4DCT scan and 20 (cases from Oxford, Cardiff, Leeds) of these form the dataset for this study. We had access to 3D-CT (mandated in NeoSCOPE protocol) and 4D-CT planning scans (optional and not mandated in the NeoSCOPE trial) along with associated quality-assured structure sets (target volumes and organs at risk - heart, lungs, spinal cord and liver). A 4D-CT PTV had already been created by the treating centre, according to the NeoSCOPE 4D-CT protocol. An experienced clinical oncology trainee also generated a 3D-CT PTV on each of the cases using the 3D-CT planning scan, according to the NeoSCOPE 3D protocol. These were approved by a consultant clinical oncologist (SG), who was quality assurance (QA) lead for the NeoSCOPE trial. As the NeoSCOPE trial protocol required a 3D-CT scan for the radiotherapy planning, it was possible to use these 3D-CT scans and compare the plans generated with the patients who also had a 4D-CT plan generated.

Physics Planning

All twenty patients had a 3D conformal plan based on 4D-CT created at the treating centre. For comparison, we created a 3D conformal plan based on the newly generated 3D-CT PTV. As NeoSCOPE was a multi-centre trial, the original 4D-CT PTV plans were created using a range of different linear accelerator machines and treatment planning systems (TPS). To account for any variation, plans optimised to 3D-CT and 4D-CT PTVs were replanned using Oncentra MasterPlan (version 4.3) for the purposes of this study. Both sets of plans used the dose volume constraints set out in the NeoSCOPE protocol (see table 1). 3D conformal RT was mandated as IMRT was not allowed in NeoSCOPE due to the concerns of pulmonary toxicity at the time of recruitment. [8]

To ensure consistency with the clinically delivered plan, the same plan parameters approved as part of the NeoSCOPE trial were used where possible, and then a physicist (AS) adjusted the plan parameters where necessary (e.g. if the original plan was generated using $5 \mathrm{~mm}$ width 
MLC leaves, some adjustments to MLC segments were required to optimise plan quality) to optimise the dose distribution (maximising the target coverage whilst minimising the OAR dose). A similar target conformality was achieved for both plans and the dose received by the surrounding OARs was assessed. All plans met NeoSCOPE dose constraints. To ensure plans were clinically acceptable, each plan was reviewed and approved by the RTTQA lead for the trial. (SG)

\begin{tabular}{|l|l|}
\hline \multicolumn{1}{|c|}{ Dose reported } & \multicolumn{1}{c|}{ Constraint } \\
\hline Total PTV volume (ccm) & - \\
\hline PTV (type B algorithm) & V95\% $>99 \%$ \\
\hline ICRU maximum dose & D1.8cc $<107 \%$ \\
\hline Combined lung V20Gy & $<25 \%$ \\
\hline Heart V25Gy & $<50 \%$ \\
\hline Heart V40Gy & $<30 \%$ \\
\hline Liver V30Gy & $<60 \%$ \\
\hline Spinal cord PRV & D0.1cc $<40 G y$ \\
\hline Table 1: Dose objectives specified within the NeoSCOPE trial &
\end{tabular}

The mean differences between the 3D and 4D plans for each of the OAR dose constraints stated in Table 1 were calculated.

\section{Statistical Analysis}

Key metrics related to the two plans were included, including reporting summary measures such as means, medians and percentiles. Comparisons were reported both in terms of statistical significance and using more intuitive measures such as the proportion of patients who would have seen a reduction in risk and the median percentage reduction in exposure.

The distributions of the variables related to the dose constraints were varied and frequently non-normal. In the interest of consistency and to ensure robust results non-parametric methods were used to assess statistical significance. More specifically, since each patient 
provided matching observations for the two plans (3D and 4D), each constraint was tested separately using the Wilcoxon Signed Rank test.

In addition, the findings were reported in terms of the reduction in estimated complication probability.

Normal Tissue Complication Probability (NTCP) Models

In an attempt to assess the clinical significance of the calculated dose volume differences between the 3D and 4D derived treatment plans, NTCP were calculated for the heart and the lung volumes within each case. The Lyman-Kutcher-Burman (LKB) model was used to predict the NTCP for the heart and the lung following radiotherapy treatment, as used often within the literature. $[10,11]$

The LKB model calculates NTCP values for different tissues using the equations and parameters included in the appendix (see appendix 2). The LKB model descibes the sigmoidal dose response observed by OARs as an error function. This function is used to calculate the probability of a specific toxicity end-point occuring, and is dependent upon the magnitude of the dose incident on the OAR, as well as the proportion of the OARs volume which is irradiated to that dose level

There are currently no well-validated LKB models for post-operative lung and heart complications, therefore we selected comparable parameters as surrogates for these endpoints. A review of the literature led to two sets of LKB parameters being chosen for the heart and the lungs to attempt to minimise any impact of the LKB model parameters. The lung models selected assess the probability of inducing grade 2 or grade 3 (or higher) radiation pneumonitis, and the heart models end points under investigation are pericardial effusion and radiation induced heart valvular dysfunction.

Along with the DVH data for the heart and lung exported from the TPS, the model parameters $n, m$ and $d 50$ were used within the LKB model to generate NTCP data for each patient.

CERR [12] was used to generate NTCP values using the DVH data and LKB parameters. CERR is an open source software environment that is based on MATLAB and can be used to evaluate treatment plans using various parameters.

Results 
Plan Statistics

The median volume of the 3D-CT PTVs was $539 \mathrm{~cm}^{3}$ compared to $391 \mathrm{~cm}^{3}$ for the 4D-CT PTVs (median difference of $148 \mathrm{~cm}^{3}$ ) giving a percentage reduction in volume of $28 \%$ for $4 D-C T$ PTVs $(p=<0.01)$.

DVH results comparing 3D-CT and 4D-CT plans for the 20 patients are described in Figure 1 and Table 2.

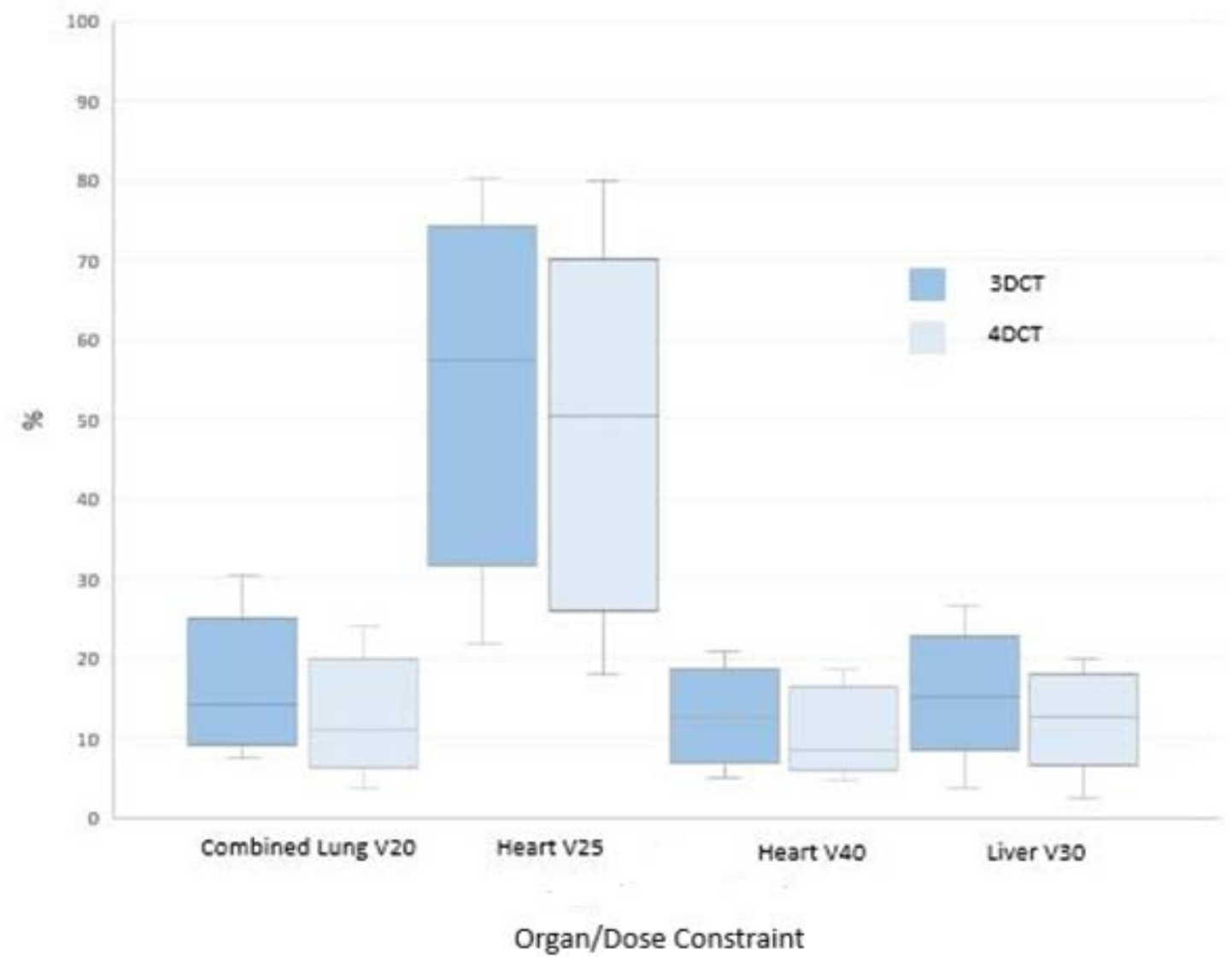

Figure 1: DVH Results for 3D-CT and 4D-CT plans

\begin{tabular}{|c|c|c|c|c|c|}
\hline \multirow{2}{*}{ Organ } & \multirow{2}{*}{ Units } & \multicolumn{5}{|c|}{ Dose } \\
\cline { 3 - 6 } & & Min & Max & Median & IQR \\
\cline { 3 - 6 } & & 2736 & 4496 & 3309 & $3188-3530$ \\
\hline Spinal cord PRV (3D-CT) & $\mathrm{cm}^{3}$ & 2635 & 4351 & 3160 & $2847-3363$ \\
\hline Spinal cord PRV (4D-CT) & $\mathrm{cm}^{3}$ & &
\end{tabular}

Table 2: Dose to Spinal Cord PRV 


\section{Comparison of Plans}

For each dose constraint a comparison was made between the plans. The results were consistent, with the 4D-CT plan resulting in significantly lower dose levels in every case, with results all significant at the $1 \%$ level or below, despite the modest sample size.

The majority of patients would have seen a reduction in the percentage of the OAR receiving a given dose. For example, the smallest improvement was observed for spinal cord PRV of which only $75 \%$ of patients would have seen a dose reduction. The expected median reduction in this case was $4 \%$. Results were much more notable for the other constraints, with $80 \%$ of the patients seeing a reduction in the volume of the heart receiving $40 \mathrm{~Gy}$. This represents a median reduction (IQR) of $23 \%$.

When the entire volume exposed is considered, all patients would have benefitted from having a $4 \mathrm{D}-\mathrm{CT}$ plan, with a $29 \%$ reduction in total integral dose. Further details can be found in Table 3.

\begin{tabular}{|c|c|c|c|c|}
\hline & $\begin{array}{c}\text { Proportion of } \\
\text { cases lower } \\
\text { for 4D (\%) }\end{array}$ & $\begin{array}{c}\text { Median } \\
\text { Reduction (\%) }\end{array}$ & IQR & \\
\hline Combined lung (\%) & $95 \%$ & $20 \%$ & $15 \%-24 \%$ & 0.001 \\
\hline Heart V25 (\%) & $95 \%$ & $10 \%$ & $5 \%-15 \%$ & 0.001 \\
\hline Heart V40 (\%) & $80 \%$ & $23 \%$ & $5 \%-34 \%$ & 0.002 \\
\hline Liver V30 (\%) & $90 \%$ & $16 \%$ & $10 \%-26 \%$ & 0.001 \\
\hline Spinal cord PRV (cGy) & $75 \%$ & $4 \%$ & $0 \%-10 \%$ & 0.005 \\
\hline Volume (ccm) & $100 \%$ & $29 \%$ & $18 \%-37 \%$ & $<0.001$ \\
\hline
\end{tabular}

Table 3: Significance testing for reduction in dose to OARs

NTCP

\begin{tabular}{|l|l|l|l|}
\hline Radiobiological & End Point & $\begin{array}{l}\text { Absolute Reduction } \\
\text { in risk of 4D vs 3D }\end{array}$ & $\begin{array}{l}\text { Relative reduction } \\
\text { in risk of 4D vs 3D }\end{array}$ \\
\hline Burman et al.[10] & $\begin{array}{l}\text { Pericarditis/pericardial } \\
\text { effusion }\end{array}$ & $0.018 \% \pm 0.001 \%$ & $33.9 \% \pm 11.6 \%$ \\
\hline
\end{tabular}




\begin{tabular}{|l|l|l|l|}
\hline Cella et al.[11] & $\begin{array}{l}\text { Radiation induced heart } \\
\text { valvular dysfunction }\end{array}$ & $1.9 \% \pm 0.39 \%$ & $95.0 \% \pm 1.7 \%$ \\
\hline Yorke et al.[13] & Lung pneumonitis (>grade 3) & $0.056 \% \pm 0.0002 \%$ & $24.1 \% \pm 8.3 \%$ \\
\hline De Jaeger et al.[14] & $\begin{array}{l}\text { Lung radiation pneumonitis: } \\
\text { grade 2, (symptoms requiring } \\
\text { steroids) or higher }\end{array}$ & $1.005 \% \pm 0.11 \%$ & $81.1 \% \pm 3.5 \%$ \\
\hline
\end{tabular}

Table 4: Absolute and relative risk reduction of heart and lung toxicity endpoints for 4D-CT and 3D-CT plans

Unsurprisingly, given the significant improvements in dose, the models all reported a reduction in risk. However, while the models agreed that there would be a positive benefit in terms of NTCP, the magnitude of the effect varies considerably, according to the endpoint under investigation.

Although absolute risk reductions are small, the relative risk reductions are significant. The relative risk of cardiac toxicity, because of using the 4D plan, is estimated at around $33 \%$ for pericarditis/pericardial effusion and $95 \%$ by radiation induced heart valvular dysfunction. Similarly for lung toxicity, the relative risk reduction to the of grade 3 lung pneumonitis is $24 \%$ while for grade 2 toxicity, a figure of $81 \%$ is obtained. Further details are found in Table 4.

In all cases the effect size is large relative to the confidence interval, indicating a real treatment effect is likely to occur and, while there is variation, the magnitude for some patients/endpoints predicting the potential for meaningful clinical benefit.

\section{Discussion}

Our study, which to the best of our knowledge is the first to study the dosimetric benefit of incorporation of 4D-CT into RT for OC, has shown a reduction of dose to heart and lungs which may reduce rates of treatment toxicity. While this study only indicated minimal absolute differences in heart and lung complication probability when comparing 3D-CT and 4D-CT target delineation methods, the significant relative improvements between the two techniques suggest the use of a 4D-CT delineation protocol and treatment technique could provide a clinically meaningful benefit compared to 3D-CT plans. In addition, there was substantial reduction in absolute volume of PTV and integral dose which would facilitate 
further reductions to OARs, even taking into consideration newer techniques such as VMAT and proton beam therapy (PBT).

\section{Post-operative Toxicity Reduction}

The Esophagectomy Complications Consensus Group (ECCG) recently showed that grade 3-5 cardiac and pulmonary complications occurred in approximately $50 \%$ of patients postoesophagectomy. The two most common toxicities were pneumonia (14.6\%) and atrial dysrhythmias (14.5\%). A significant proportion of patients received nCRT [2].

These rates of severe complications are unacceptably high. In the UK, there remains a reticence in adopting $\mathrm{nCRT}$ for OC compared to other developed countries due in part to the concern over post-operative toxicities. $[3,4]$ While perioperative care continues to improve with the adoption of programmes such as Enhanced Recovery after Surgery (ERAS)[15], radiation therapy must also adopt new strategies to optimise treatment to minimise toxicity from neo-adjuvant treatment.

Lung

Wang et al showed that mean lung dose is strongly associated with post-operative pulmonary complications. [16] Recently published retrospective data comparing 3DRT, IMRT and PBT showed that post-oesophagectomy lung complications can be reduced by using techniques that spare dose to lung pre-operatively. [17] Our work has shown 4D-CT will reduce combined lung dose by around $20 \%$ with a probability of grade 3 pneumonitis reduction of $24.1 \%$. Although there is no randomised control data, we can infer that the use of 4D-CT may contribute to a reduction in post-operative lung toxicity.

Heart

Lin et al showed that there was a significantly improved overall survival (OS) and locoregional control when IMRT was used to treat patients with OC compared to 3D-CRT, with an excess of non-cancer related deaths in the 3D-CRT group compared to IMRT. This was postulated to be due to an excess of cardiac related deaths in the 3D-CRT group, likely related to the toxic sequelae of thoracic radiotherapy with IMRT resulting in lower cardiac doses. These effects were seen within 2 years of CRT. In a separate review of postoesophagectomy complications, Lin et al showed that more conformal radiation techniques such as IMRT and PBT resulted in significantly less cardiac complications. $[17,18]$ Mukherjee 
et al demonstrated that a significant dose of radiation during oesophageal chemo radiotherapy correlates to a reduction in the cardiac ejection fraction.[19] There is emerging evidence that a seemingly small dose to specific cardiac substructures such as the sinoatrial node can result in a higher incidence of acute arrhythmias. [20] Our work shows a mean reduction of heart V40 of $23 \%$, with improvements seen in $80 \%$ of the cases. These retrospective data clearly support the need of minimising pre-operative dose to the heart to optimise post-operative and long term outcomes.

\section{Emerging Adjuvant Treatments}

The positive findings of the PACIFIC trial of adjuvant immunotherapy (Durvalumab) in lung cancer may herald a significant shift in approach to adjuvant therapies post CRT for several tumour sites [21]. Early clinical data of adjuvant Durvalumab in OC has shown similar promise. [22] The PACIFIC trial reported a pneumonitis rate of $33.9 \%$ in the experimental arm. [21] This is likely to be a treatment-limiting toxicity for a significant proportion of patients. In our study, the use of 4D-CT led to a relative risk reduction of $81 \%$ of grade 2 pneumonitis and a reduction of $24.1 \%$ for grade 3 pneumonitis. Adopting strategies such as 4D-CT to minimise dose to lung will help ensure the greatest number of patients will be able to benefit from these emerging treatments.

\section{Late Toxicity Reduction}

OC survival rates for 10 years or more has improved from $4 \%$ to $12 \%$ in the last 40 years.[1] The CROSS trial, a study of CRT followed by surgery, demonstrated a median overall survival of 84.1 months and 48.6 months for patients with squamous and adenocarcinoma of the oesophagus respectively.[23] As patients live longer following CRT, it becomes increasingly important to minimise dose in order to limit the long-term side effects causing morbidity and mortality after treatment. Long term cardiac and pulmonary toxicities following radiation exposure are well established. Darby et al elegantly demonstrated the risk of major coronary events is increased by $7.4 \%$ per Gy with the effect starting only a few years after radiation exposure in breast cancer patients. [24] In lung cancer, dose to lung and heart has been show to clearly impact on long term survival outcomes. $[25,26]$ Our work has shown how the 
incorporation of $4 \mathrm{D}-\mathrm{CT}$ has a role to play in reducing dose to OARs by decreasing the high dose region (e.g. V40 Heart) as well as a reduction in integral dose.

\section{Limitations}

Despite the trial being multi-centre, numbers of $4 \mathrm{D}$ cases were small. This was due to technical factors such as obtaining complete datasets for our analysis and trial factors as 4DCT was not mandated and was still relatively new to the UK oesophageal RT community at the time [27]. Additionally, we were unable to access baseline demographic data including smoking and cardiac history. However, we do not believe this detracts from the overall findings of this 'proof of concept' study, as this was purely a dose distribution and comparison study, the findings of which are independent of patient demographics.

At the time of the study, concerns regarding lung toxicity led to the decision to avoid IMRT/VMAT techniques. This study has therefore limited the comparison to 3D-CT and 4D-CT PTV volume using 3D conformal planning. Increasingly, although not exclusively, oesophageal radiotherapy is being delivered via IMRT/VMAT. [27] It is unclear if the magnitude of benefit seen in our study will be maintained when using IMRT/VMAT. Despite this limitation, this work still shows how absolute PTV volume and integral dose is reduced using 4D-CT which will undoubtedly give dosimetric advantages irrespective of radiation technique.

LKB models have historically been widely used but they have been criticised for oversimplifying dose-volume effects and failing to consider complex biological processes and interactions thus affecting its predictive power. Increasingly, data-driven logistic regressionbased models are being used and may more accurately predict NTCP, particularly in larger cohorts of patients. $[11,28]$ In addition, there is a lack of validated NTCP models looking specifically at post-oesophagectomy toxicity with several currently in development. [29] Predicted NTCP values provide a broad indication of toxicity rates for purposes of this study but caution is needed when translating this into the clinical setting.

\section{Recommendations and Future work}

The use of 4D-CT planning scans alongside precision radiotherapy techniques (IMRT and VMAT) will allow for a further improvement in the therapeutic ratio as both methods allow for a reduction in post-operative toxicities, late effects and may maximise the potential use 
of new adjuvant treatments. 4D-CT allows individualised margins, which are smaller than the traditional population-based margins, with reduction in dose to the OARs. We believe that benefits of this study are widely applicable and reproducible as 4D-CT is now broadly available with a recent survey showing $71 \%$ of UK cancer centres now use 4D-CT. [27] Further work should quantify the magnitude of benefit when using IMRT/VMAT with 4D-CT compared to 3D-CT planning scans.

Future work should also explore the role of PBT in further reducing the dose to OARs. Proton's characteristic Bragg peak allows minimal dose to be deposited to tissues distal to the target volume. Given the oesophagus' proximity to organs such as the heart and lung, PBT has the potential to further improve the therapeutic ratio. Planning studies comparing modern PBT pencil beam scanning (PBS) technology to precision photon radiotherapy techniques (IMRT and VMAT) have shown dosimetric advantage of PBT with potential reduction of lung and heart dose.[30,31] It is important to note that the use of 4D-CT in PBT to the thoracic region is already prerequisite in the majority of published literature. [32] This is due to the sensitivity of PBT dose distribution to intra-fraction motion and tissue heterogeneity, which necessitates the use of $4 \mathrm{D}-\mathrm{CT}$ to create robust treatment plans.

In summary, 4D-CT, along with other precision radiotherapy techniques, play a vital role in maximising the potential of CRT in oesophageal cancer. We recommend that, where possible, all patients undergoing chemo-radiotherapy for potentially curable gastrooesophageal junction tumours should receive 4D-CT planning scans to allow a reduction in dose to the OARs in order to reduce morbidity associated with treatment and potentially improve survival outcomes. 


\section{Appendix 1: NeoSCOPE Outlining Protocol}

Outlining Protocol for Volume Delineation (as used in the NeoSCOPE Trial):

- The GTV was outlined using diagnostic imaging (CT, PET and EUS) on the 3D scan as well as the maximum inspiration and expiration phases of the 4D scan.

- CTV A on the 3D scan was created by manually extending the GTV motion $2 \mathrm{~cm}$ proximally and distally (or until the gastro-oesophageal junction). If the superior extent of the tumour was defined by nodal disease, then the CTV A extension from the node was $1 \mathrm{~cm}$.

- $\quad$ CTV B was formed by CTV A being copied and a $1 \mathrm{~cm}$ circumferential margin was added. This was edited for bone, lung, pericardium and the great vessels. For lower oesophageal tumours, below the GOJ CTV B was extended for $2 \mathrm{~cm}$ to include the atrisk lymph nodes regions (lesser curvature of the stomach, left gastric artery and coeliac region). CTVB 3D was copied and labelled CTVB maximum expiration and CTV $B$ maximum inspiration. These were edited on the respective sequences as on the $3 D$ scan.

- The 3D GTV was combined with the 4D GTVs. There are two methods for this:

1. On the 4D data sets the maximum and minimum phases of motion are identified as well as the phase that represents the time weighted average (mid phase). On each phase the GTV, CTVA and CTVB are generated (as detailed below). The ITV is formed by a composite of CTVB volumes and edited to account for any additional motion seen from all the other 4DCT phases.

2. Or: the GTV is contoured on the 3D scan. The $4 D$ CT scan is reconstructed on 10 respiratory phases. The inhale and exhale phases are identified (usually between 0 and $50 \%$, respectively). All reconstructions are reviewed to ensure that the phases represent the extremes. The GTV is contoured in extreme phases and then the GTV3D GTVMaxIn and GTV MaxEx is combined to give GTV motion. This volume is checked on all phases of respiration to ensure that all areas are covered. On the 3D contrast scan create CTVA3D and CTVB3D using the GTV motion. To obtain the ITV make a copy of CTVB3D and name it CTVBMaxIn and edit it on the maximum inhale scan. Copy CTVB3D and label it CTVBMaxln and edit this on the maximum exhale scan. ITV is formed by combining CTVBMaxIn and CTVBMaxEx.

- The ITV had a $5 \mathrm{~mm}$ margin added in all directions to account for set up error, this was labelled the PTV. 
Appendix 2: LKB model and parameters

$$
\begin{aligned}
& N T C P=\frac{1}{\sqrt{2 \pi}} \int_{-\infty}^{t} e^{-t^{2} / 2} d t \\
& t=\frac{D-T D_{50}(v)}{m T D_{50}(v)} \\
& T D_{50}(v)=T D_{50}(l) v^{-n}
\end{aligned}
$$

Where $D$ represents the Equivalent Uniform Dose (EUD) delivered to the organ (that results in the same NTCP as the planned non-uniform dose distribution), $T D_{50}$ is the tolerance dose to the whole organ which for a given partial volume fraction $v$, results in a $50 \%$ complication risk, $m$ represents the slope of the NTCP dose-response curve, and $n$ represents the volume effect of the organ being assessed which can range from 0 to 1 . The parameter quantifies the serial or parallel nature of a given OAR, with smaller values relating to an organ that exhibits a serial dose response, and larger values reflecting more of a parallel response.

\begin{tabular}{|l|l|l|l|}
\hline Organ & LKB Model Parameters & End Point & Reference \\
\hline Heart & $\mathrm{a}=2.857, \mathrm{~m}=0.1, \mathrm{TD}=48 \mathrm{~Gy}$ & $\begin{array}{l}\text { Pericarditis/pericardial } \\
\text { effusion }\end{array}$ & Burman et al[10] \\
\hline Heart & $\mathrm{a}=6.25, \mathrm{~m}=0.67, \mathrm{TD}=32.8 \mathrm{~Gy}$ & $\begin{array}{l}\text { Radiation induced heart } \\
\text { valvular dysfunction }\end{array}$ & Cella et al[11] \\
\hline Lung & $\mathrm{a}=1.149, \mathrm{~m}=0.18, \mathrm{TD}=24.5 \mathrm{~Gy}$ & $\begin{array}{l}\text { Lung pneumonitis (>grade } \\
3 \text { ) }\end{array}$ & Yorke et al. [13] \\
\hline Lung & $\mathrm{a}=1, \mathrm{~m}=0.45, \mathrm{TD}=29.2 \mathrm{~Gy}$ & $\begin{array}{l}\text { Lung radiation } \\
\text { pneumonitis: grade 2, } \\
\text { (symptoms requiring } \\
\text { steroids) or higher }\end{array}$ & De Jaeger et al [14] \\
& & & \\
\hline
\end{tabular}




\section{References}

1. Cancer Research UK Statistics. https://www.cancerresearchuk.org/aboutcancer/oesophageal-cancer/survival (accessed 19th November 2019).

2. Low, D.E., M.K. Kuppusamy, D. Alderson, I. Cecconello, A.C. Chang, G. Darling, et al., Benchmarking Complications Associated with Esophagectomy. Ann Surg, 2019. 269(2): p. 291-298.

3. National Oesophago-Gastric Audit (NOGCA) Annual Report 2018. Retrieved from https://www.nogca.org.uk/reports/2018-annual-report/.

4. Bowden, C. and S. Gwynne, Neoadjuvant chemoradiotherapy in resectable oesophageal cancer patients: UK practice. European Journal of Surgical Oncology, 2016. 42(11): p. S225.

5. Cole, A.J., J.M. O'Hare, S.J. McMahon, C.K. McGarry, K.T. Butterworth, J. McAleese, et al., Investigating the potential impact of four-dimensional computed tomography (4DCT) on toxicity, outcomes and dose escalation for radical lung cancer radiotherapy. Clin Oncol ( $\mathrm{R}$ Coll Radiol), 2014. 26(3): p. 142-50.

6. Yamashita, H., S. Kida, A. Sakumi, A. Haga, S. Ito, T. Onoe, et al., Four-dimensional measurement of the displacement of internal fiducial markers during 320-multislice computed tomography scanning of thoracic esophageal cancer. Int J Radiat Oncol Biol Phys, 2011. 79(2): p. 588-95.

7. Zhao, K.L., Z. Liao, M.K. Bucci, R. Komaki, J.D. Cox, Z.H. Yu, et al., Evaluation of respiratoryinduced target motion for esophageal tumors at the gastroesophageal junction. Radiother Oncol, 2007. 84(3): p. 283-9.

8. Mukherjee, S., C.N. Hurt, S. Gwynne, D. Sebag-Montefiore, G. Radhakrishna, S. Gollins, et al., NEOSCOPE: A randomised phase II study of induction chemotherapy followed by oxaliplatin/capecitabine or carboplatin/paclitaxel based pre-operative chemoradiation for resectable oesophageal adenocarcinoma. European journal of cancer (Oxford, England : 1990), 2017. 74: p. 38-46.

9. Mukherjee, S., C.N. Hurt, S. Gwynne, A. Bateman, S. Gollins, G. Radhakrishna, et al., NEOSCOPE: a randomised Phase II study of induction chemotherapy followed by either oxaliplatin/capecitabine or paclitaxel/carboplatin based chemoradiation as pre-operative regimen for resectable oesophageal adenocarcinoma. BMC Cancer, 2015. 15(1): p. 48.

10. Kutcher, G.J. and C. Burman, Calculation of complication probability factors for non-uniform normal tissue irradiation: The effective volume method gerald. International Journal of Radiation Oncology*Biology*Physics, 1989. 16(6): p. 1623-1630.

11. Cella, L., G. Palma, J.O. Deasy, J.H. Oh, R. Liuzzi, V. D'Avino, et al., Complication probability models for radiation-induced heart valvular dysfunction: do heart-lung interactions play a role? PloS one, 2014. 9(10): p. e111753-e111753.

12. Deasy, J.O., A.I. Blanco, and V.H. Clark, CERR: a computational environment for radiotherapy research. Med Phys, 2003. 30(5): p. 979-85.

13. Yorke, E.D., A. Jackson, K.E. Rosenzweig, S.A. Merrick, D. Gabrys, E.S. Venkatraman, et al., Dose-volume factors contributing to the incidence of radiation pneumonitis in non-small-cell lung cancer patients treated with three-dimensional conformal radiation therapy. International Journal of Radiation Oncology*Biology*Physics, 2002. 54(2): p. 329-339.

14. De Jaeger, K., M.S. Hoogeman, M. Engelsman, Y. Seppenwoolde, E.M.F. Damen, B.J. Mijnheer, et al., Incorporating an improved dose-calculation algorithm in conformal radiotherapy of lung cancer: re-evaluation of dose in normal lung tissue. Radiotherapy and Oncology, 2003. 69(1): p. 1-10.

15. Low, D.E., W. Allum, G. De Manzoni, L. Ferri, A. Immanuel, M. Kuppusamy, et al., Guidelines for Perioperative Care in Esophagectomy: Enhanced Recovery After Surgery (ERAS((R))) Society Recommendations. World J Surg, 2019. 43(2): p. 299-330. 
16. Wang, J., C. Wei, S.L. Tucker, B. Myles, M. Palmer, W.L. Hofstetter, et al., Predictors of postoperative complications after trimodality therapy for esophageal cancer. Int J Radiat Oncol Biol Phys, 2013. 86(5): p. 885-91.

17. Lin, S.H., K.W. Merrell, J. Shen, V. Verma, A.M. Correa, L. Wang, et al., Multi-institutional analysis of radiation modality use and postoperative outcomes of neoadjuvant chemoradiation for esophageal cancer. Radiotherapy and Oncology, 2017. 123(3): p. 376381.

18. Lin, S.H., L. Wang, B. Myles, P.F. Thall, W.L. Hofstetter, S.G. Swisher, et al., Propensity Scorebased Comparison of Long-term Outcomes With 3-Dimensional Conformal Radiotherapy vs Intensity-Modulated Radiotherapy for Esophageal Cancer. International Journal of Radiation Oncology*Biology*Physics, 2012. 84(5): p. 1078-1085.

19. Mukherjee, S., D. Aston, M. Minett, A.E. Brewster, and T.D.L. Crosby, The Significance of Cardiac Doses Received During Chemoradiation of Oesophageal and Gastro-oesophageal Junctional Cancers. Clinical Oncology, 2003. 15(3): p. 115-120.

20. Chen, V.E., A.J. Song, M. Werner-Wasik, G. Owen, P. Lakhani, and B. Lu, Effect of Radiation Dose to Cardiac Substructures on the Acute Development of New Arrhythmias Following Conventionally Fractionated Radiation Treatment to the Lung. International Journal of Radiation Oncology • Biology • Physics, 2019. 105(1): p. E500.

21. Antonia, S.J., A. Villegas, D. Daniel, D. Vicente, S. Murakami, R. Hui, et al., Overall Survival with Durvalumab after Chemoradiotherapy in Stage III NSCLC. N Engl J Med, 2018. 379(24): p. 2342-2350.

22. Mamdani, H., B.J. Schneider, L.I. Abushahin, T.J. Birdas, K. Kesler, A. Lee, et al., Safety and efficacy of durvalumab following trimodality therapy for locally advanced esophageal and GEJ adenocarcinoma: Early efficacy results from Big Ten Cancer Research Consortium study. Journal of Clinical Oncology, 2019. 37(4_suppl): p. 5-5.

23. Shapiro, J., J.J.B. van Lanschot, M.C.C.M. Hulshof, P. van Hagen, M.I. van Berge Henegouwen, B.P.L. Wijnhoven, et al., Neoadjuvant chemoradiotherapy plus surgery versus surgery alone for oesophageal or junctional cancer (CROSS): long-term results of a randomised controlled trial. The Lancet Oncology, 2015. 16(9): p. 1090-1098.

24. Darby, S.C., M. Ewertz, P. McGale, A.M. Bennet, U. Blom-Goldman, D. Bronnum, et al., Risk of ischemic heart disease in women after radiotherapy for breast cancer. N Engl J Med, 2013. 368(11): p. 987-98.

25. Speirs, C.K., T.A. DeWees, S. Rehman, A. Molotievschi, M.A. Velez, D. Mullen, et al., Heart Dose Is an Independent Dosimetric Predictor of Overall Survival in Locally Advanced NonSmall Cell Lung Cancer. J Thorac Oncol, 2017. 12(2): p. 293-301.

26. McWilliam, A., J. Kennedy, C. Hodgson, E. Vasquez Osorio, C. Faivre-Finn, and M. van Herk, Radiation dose to heart base linked with poorer survival in lung cancer patients. Eur I Cancer, 2017. 85: p. 106-113.

27. Gwynne, S., E. Higgins, A. Poon King, G. Radhakrishna, L. Wills, S. Mukherjee, et al., Driving developments in UK oesophageal radiotherapy through the SCOPE trials. Radiation Oncology, 2019. 14(1): p. 26.

28. Xu, C.J., A. van der Schaaf, C. Schilstra, J.A. Langendijk, and A.A. van't Veld, Impact of statistical learning methods on the predictive power of multivariate normal tissue complication probability models. Int J Radiat Oncol Biol Phys, 2012. 82(4): p. e677-84.

29. Thomas, M., G. Defraene, M. Lambrecht, W. Deng, J. Moons, P. Nafteux, et al., NTCP model for postoperative complications and one-year mortality after trimodality treatment in oesophageal cancer. Radiotherapy and Oncology, 2019. 141: p. 33-40.

30. Hirano, Y., M. Onozawa, H. Hojo, A. Motegi, S. Zenda, K. Hotta, et al., Dosimetric comparison between proton beam therapy and photon radiation therapy for locally advanced esophageal squamous cell carcinoma. Radiat Oncol, 2018. 13(1): p. 23. 
31. Warren, S., M. Partridge, A. Bolsi, A.J. Lomax, C. Hurt, T. Crosby, et al., An Analysis of Plan Robustness for Esophageal Tumors: Comparing Volumetric Modulated Arc Therapy Plans and Spot Scanning Proton Planning. International journal of radiation oncology, biology, physics, 2016. 95(1): p. 199-207.

32. Zhang, M., W. Zou, and B.-K.K. Teo, Image guidance in proton therapy for lung cancer. Translational lung cancer research, 2018. 7(2): p. 160-170. 